\title{
Outcome of Children Admitted With SARS-CoV-2 Infection: Experiences From a Pediatric Public Hospital
}

\author{
Sudha Rao, Vrushabh Gavali, Shakuntala S Prabhu, Radhika Mathur, Larissa Robert Dabre, Sanjay B \\ Prabhu and Minnie Bodhanwala \\ From Department of Pediatrics, Bai Jerbai Wadia Hospital for Children, Mumbai, Maharashtra, India.
}

\author{
Correspondence to: Dr. Sudha Rao, \\ Professor and, Head Department of \\ Pediatrics, Bai Jerbai Wadia Hospital \\ for Children, Acharya Dhonde Marg, \\ Parel, Mumbai, India. \\ c_sudha@hotmail.com \\ Received: September 17, 2020; \\ Initial review: October 5, 2020; \\ Accepted: January 10, 2021.
}

\begin{abstract}
Objective: To study clinical characteristics and outcome of children with admitted to a paediatric hospital in Mumbai, India. Method: Review of medical records of 969 children admitted between 19 March and 7 August, 2020, to assess the clinico-demographic characteristics, disease severity and factors predicting outcome in COVID-19 children. Variables were compared between children who were previously healthy (Group I) and those with co-morbidity (Group II). Results: 123 (71 boys) children with median (IQR) age of 3 (0.76) years were admitted, of which $47(38 \%)$ had co-morbidities. $39(32 \%)$ children required intensive care and $14(11.4 \%)$ died. Male sex, respiratory manifestation, oxygen saturation $<94 \%$ at admission, mechanical ventilation, inotrope, hospital stay of $<10$ days were independent predictors of mortality. Oxygen saturation $<94 \%$ at admission (OR $35.9,95 \% \mathrm{Cl}$ 1.5-856) and hospital stay $<10$ days (OR 9.1, 95\% Cl 1.04-99.1) were significant. Conclusion: COVID-19 in children with co-morbidities causes severe disease. Association of mortality with oxygen saturation by pulse oximeter $<94 \%$ on admission, and hospital stay $<10$ days, needs further evaluation.
\end{abstract}

Keywords: Co-morbidities, Mortality, Multisystem inflammatory syndrome in children (MIS-C), Prognosis.

Published online: January 11, 2021; PII: S097475591600280

S evere acute respiratory syndrome coronavirus 2 (SARS-CoV-2) infection, leading to COVID-19 disease pandemic, has spread all over India and the world. Mumbai Metropolitan Region (MMR) and the City of Mumbai is the worst affected hotspot in India. Our center, a tertiary care, public, specialist hospital, received many children with SARS-CoV-2 from March 19, 2020. Many vulnerable children with co-morbid conditions like heart disease, malnutrition, malignancy, diabetes, chronic kidney disorder, etc. also presented for acute inter current emergencies. This retrospective study presents the demographic, clinical characteristics, treatment and, outcome, care of neonates and children with SARS-CoV-2 positivity from our center.

\section{METHOD}

Retrospective medical record review of all children admitted to the hospital between March 19, 2020 and August 7, 2020 was done. Approval of institutional ethics committee obtained. All children with reverse transcriptase-polymerase chain reaction (RT-PCR) positive for SARS-CoV-2 were studied.

As per institutional protocol, derived from national guidelines [1], every child requiring admission was tested by RT-PCR for SARS-CoV-2 from an Indian Council of
Medical Research (ICMR) recognized laboratory. Children who tested positive were admitted to the isolation ward, specially created as per national guidelines [2]. Historical details and pre-existing comorbidities were recorded. COVID-19 disease characterization was done as per guidelines [1]. Multisystem inflammatory syndrome in children (MISC) and Kawasaki disease (KD) were defined as per standard definition $[3,4]$. Institutional protocol of care created based on ICMR /GOI recommendations was followed. Some cases of MIS-C/KD included in this study have been previously published [5].

Laboratory investigations and imaging studies were carried as necessary. Therapeutic principles included general supportive therapy, active control of fever, respiratory support with oxygen and/or ventilation as necessary, vasoactive drugs in shock, and active monitoring of organ system dysfunctions. Remdesivir was given to children above 12 years of age with COVID19 pneumonia. Younger children received it on compassionate grounds with risk explained and an informed consent taken. Intravenous immunoglobulin, pulse methyl-prednisolone, and anticoagulation with low-molecular-weight heparin were used as per protocol. Repeat testing for SARS-CoV-2 PCR and discharge 
criteria were followed as per guidelines [1]. Time taken to PCR negativity and duration of hospital stay was noted. Treatment outcomes were defined as discharged or died.

SARS-CoV-2 positive children in this cohort were classified into Group I comprising of previously healthy children, and Group II with children having comorbidities like heart disease, diabetes, malignancy, malnutrition, renal, hepatobiliary, neurological, surgical/ orthopedic conditions, etc. Variables were compared between the groups.

Statistical analyses: Data was entered in MS Excel, and coded and analyzed in statistical software STATA, version 10.1 (Stata Corp.). Pearson Chi-square test was used for assessing significance of association between outcome (mortality/discharge) and exposure variables/ predictors. Binomial test for difference in proportions was also used to compare proportions in sub-groups or categories in two groups. Student t-test or MannWhitney test was performed to assess significance of difference in means or medians in two independent groups. Binary multiple logistic regression model was applied to identify predictors of mortality accounting for the role of other factors, wherein adjusted odds ratio (OR) and $95 \%$ Confidence Intervals (CI) were estimated. A $P$ value of $<0.05$ was considered statistically significant for all the comparisons.

\section{RESULTS}

Of 969 children admitted during the study period, 123 $(12.8 \%)$ tested positive for SARS-CoV-2 including 16 (13\%) extramural neonates. Five $(4.1 \%)$ had a history of travel. The median (IQR) age at presentation was 3 (0.76.0) year with a male: female ratio of 1.36 (Table I).

Seventy six (62\%) children belonged to Group I and 47 (38\%) Group II. Distribution of underlying illness in Group II is shown in Fig. 1. Children with severe COVID19 had underlying hemato-oncological, renal and cardiac disorders. Children in Group I presented at a younger age than those in Group II [median (IQR) 1.7 (10.5-5.25) vs. 4 (0.8-9) years; $P=0.052] ; 27$ (22\%) children were asymptomatic. Fever in $24(20 \%)$ or respiratory symptoms in $30(24 \%)$ children were common presenting symptoms, and seizures in $13(10 \%)$ and gastrointestinal symptoms in $15(12.2 \%)$ children were the atypical presentations. Six (5\%) children presented with injuries like head injury / limb fracture. Interestingly, severe COVID-19 disease was seen more in Group II whereas MIS-C/KD was more in Group I (Table I).

On admission, mean (SD) pulse oximeter saturation (SpO2) and the blood neutrophil: lymphocyte ratio was lower, respectively in Group I than Group II [94.7 (7.1) vs
Table I Baseline Characteristics, Clinical Profile and Outcome in Children With SARS-CoV-2 Infection $(N=123)$

\begin{tabular}{|c|c|c|c|}
\hline & All children & $\begin{array}{l}\text { Previously } \\
\text { healthy } \\
(n=76)\end{array}$ & $\begin{array}{r}\text { With co- } \\
\text { morbidity } \\
(n=47)\end{array}$ \\
\hline Male & $71(57.7)$ & $43(56.6)$ & $28(59.6)$ \\
\hline \multicolumn{4}{|l|}{ Age wise distribution } \\
\hline$<1$ mo of age & $16(13.0)$ & $12(15.8)$ & $4(8.5)$ \\
\hline $1 \mathrm{mo}-1 \mathrm{y}$ & $31(25.2)$ & $20(26.3)$ & $11(23.4)$ \\
\hline $1 y-5 y$ & $39(31.7)$ & $25(32.9)$ & $14(29.8)$ \\
\hline $5-10 y$ & $26(21.1)$ & $17(22.4)$ & $9(19.2)$ \\
\hline$>10 \mathrm{y}^{c}$ & $11(8.9)$ & $2(2.6)$ & $9(19.2)$ \\
\hline \multicolumn{4}{|l|}{ Symptoms at presentation } \\
\hline Asymptomatic $^{c}$ & $27(21.9)$ & $8(13.2)$ & $19(36.2)$ \\
\hline Fever & $24(19.5)$ & $16(21.0)$ & $8(17.0)$ \\
\hline Upper respiratory & $5(4.1)$ & $3(3.9)$ & $2(4.3)$ \\
\hline Lower respiratory & $25(20.3)$ & $18(23.7)$ & $7(14.9)$ \\
\hline Gastrointestinal & $15(12.2)$ & $12(15.8)$ & $3(6.4)$ \\
\hline Seizures ${ }^{b}$ & $13(10.6)$ & $12(15.8)$ & $1(2.1)$ \\
\hline Others $^{b}$ & $14(11.4)$ & $5(6.6)$ & $9(19.2)$ \\
\hline Radiology & $n=114$ & $n=73$ & $n=41$ \\
\hline Abnormal $X$-ray chest & $23(20)$ & $16(22.0)$ & $7(27.7)$ \\
\hline \multicolumn{4}{|l|}{ Disease severity } \\
\hline Mild $^{d}$ & $54(43.9)$ & $50(65.8)$ & $4(8.5)$ \\
\hline Moderate $^{d}$ & $26(21.1)$ & $4(5.3)$ & $22(46.8)$ \\
\hline Severe $^{b}$ & $32(26.0)$ & $14(18.4)$ & $18(38.3)$ \\
\hline MIS-C/KD ${ }^{c}$ & $11(8.9)$ & $8(10.5)$ & $3(6.4)$ \\
\hline Need for intensive care & $39(31.7)$ & $24(31.6)$ & $15(31.9)$ \\
\hline \multicolumn{4}{|l|}{ Respiratory support } \\
\hline Only oxygen & $20(16.3)$ & $13(17.1)$ & $7(14.9)$ \\
\hline Non-invasive ventilation & $6(4.9)$ & $5(6.7)$ & $1(2.1)$ \\
\hline Invasive ventilation & $13(10.6)$ & $8(10.5)$ & $5(10.6)$ \\
\hline Vasoactive drugs used & $17(13.8)$ & $11(14.5)$ & $6(12.8)$ \\
\hline \multicolumn{4}{|l|}{ Outcome } \\
\hline Death & $14(11.4)$ & $6(7.9)$ & $8(17.0)$ \\
\hline Discharge & $105(85.4)$ & $68(89.5)$ & $37(78.7)$ \\
\hline Still admitted & $4(4)$ & $2(2.6)$ & $2(4.25)$ \\
\hline
\end{tabular}

Values in no. (\%) except amedian (IQR). For comparison between groups $\quad{ }^{b} P<0.05, \quad{ }^{c} P<0.01, \quad{ }^{d} P<0.001 . \quad$ MIS-C/KD-Multisystem Inflammatory Syndrome in Children/Kawasaki disease.

96.5 (6.1); $P=0.09$ ] and [2.6 (3.6) vs 4.5 (5.1); $P=0.09$ ]. Chest radiograph was done in $114(93 \%)$ cases, it was abnormal in 23 (20\%) with bilateral haziness, consolidation and pleural effusion being the common abnormalities.

Eighty four $(68.3 \%)$ children did not require respiratory support. More number of children in Group I $(n=13 / 19)$ required ventilator care. Vasoactive drugs required in $17(14 \%)$ cases and 11 were from Group I 


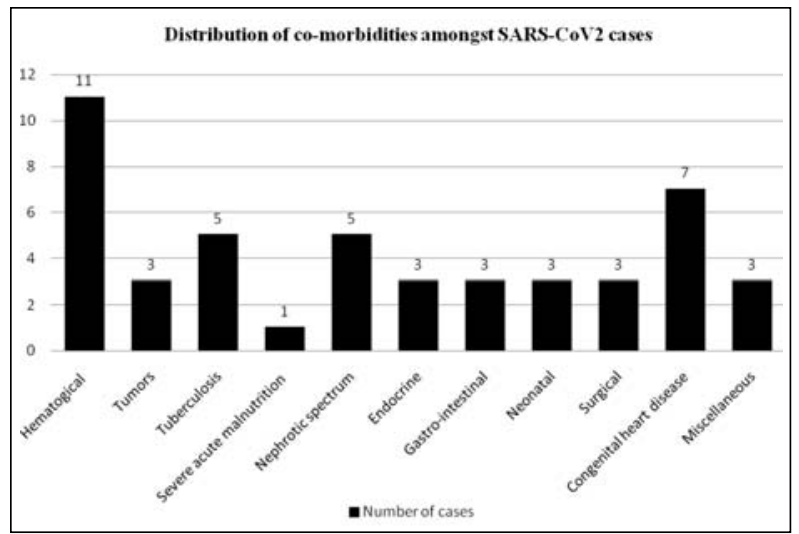

Fig. 1 Distribution of co-morbidities amongst children with SARS-CoV-2 infection.

(Table I). Severe COVID-19 pneumonia $(n=10 / 39)$, circulatory collapse $(n=5 / 39)$, MIS-C/KD $(n=8 / 39)$, worsening of underlying disease $(n=16 / 39)$ as indications, 39 (32\%) children needed intensive care, which was similar in Groups I and II. Left ventricular dysfunction $(n=6)$, dilatation of coronaries $(n=2)$ were the echocardiography findings in eight children with MIS-C/KD; 4 (50\%) children received IVIG within 48 hours. Remdesivir was given to two children with severe COVID-19 pneumonia.

While compiling the study, 4 children were still admitted. The median duration of PCR negativity was 5 days (range, 3-15 days). 105 (88\%) patients were discharged. The median duration of PCR negativity was 5 days (range, 3-15 days); 105 (88\%) patients were discharged. The median (range) length of hospital stay was 9 days (4-17 days), which did not differ significantly between Groups I and II (Table I).

There were 14 (11.4\%) deaths of which 3 (21.5\%) were neonates. Four children in Group II who died had underlying malignancy. Male sex, $\mathrm{SpO} 2<94 \%$ at admission, abnormal chest $X$-ray, need for respiratory support, need for vasoactive support, need for intensive care and the duration of hospital stay were predictors of mortality on univariate analysis (Table II). $\mathrm{SpO} 2<94 \%$ at admission [OR (95\% CI) 9.1 (1.04-99.1); $P=0.04$ ] and hospital stay of less than 9 days [OR $(95 \%$ CI) 35.9 (1.5$856.0) ; \quad P=0.02$ ] were predictors of mortality on regression analysis.

\section{DISCUSSION}

The study highlights the clinical characteristics, disease progression, and outcome of 123 children admitted with COVID-19. As admitted children were enrolled, the data likely represents individuals from the moderate-to-severe end of the disease spectrum.
The proportion of previously healthy children was $62 \%$. In a study from Columbia Pediatric COVID-19 management group co-morbidities defined as obesity, asthma, infancy or immune suppression were studied [6].Recent data from US studied chronic lung disease, cardiovascular disease and immune suppression as the common co-morbidities [7].Twenty seven (21.7\%) children were asymptomatic comparable to the metaanalysis where $23 \%$ were asymptomatic [8]. Initial studies from China reported $4.1-50 \%$ cases to be asymptomatic, while $58 \%$ were asymptomatic in a study from Pune $[9,10]$.Fever and respiratory symptoms were the common presenting symptoms as also found by others [8-10]. Atypical presentations like seizures (10.6\%), gastrointestinal symptoms (12.2\%) were more common in this series as compared to other studies[7,10,11]. More children in our cohort had severe disease as compared to only $1 \%$ as reported in recent retrospective study from China[12].Children with underlying hemato-oncological, renal or cardiac disorders had severe disease. Interestingly, the immune response of COVID-19, the MIS-C/KD was found more in Group I than Group II. This has been reported in other studies also[5,13].Presence of comorbidity dysregulates or blunts the immunological host responses causing severe infection but a hyper-inflammatory immune response like MIS-C/KD is not seen.

Table II Predictors of Outcome in Pediatric Inpatients With SARS-CoV-2 Infection $(N=119)$

\begin{tabular}{|c|c|c|c|}
\hline Factors & $\begin{array}{l}\text { Death } \\
(n=14)\end{array}$ & $\begin{array}{l}\text { Discharge } \\
(n=105)\end{array}$ & $\begin{array}{r}\text { OR } \\
95 \% C I\end{array}$ \\
\hline Age at presentation $\geq 3 \mathrm{y}$ & $7(50)$ & $56(88.9)$ & $\begin{array}{r}0.88 \\
(0.24-3.15)\end{array}$ \\
\hline Male $\operatorname{sex}^{a}$ & $11(78.6)$ & $58(55.2)$ & $\begin{array}{r}4.52 \\
(1.1-26.4)\end{array}$ \\
\hline Asymptomatic & 0 & $27(25.7)$ & - \\
\hline Respiratory symptoms ${ }^{a}$ & $10(71.4)$ & $41(39.1)$ & - \\
\hline $\begin{array}{l}\text { Normal } X \text {-ray chest, }{ }^{b} \\
n=110\end{array}$ & $5(35.7)$ & $82(80)$ & $\begin{array}{r}7.2 \\
(1.9-29.7)\end{array}$ \\
\hline $\mathrm{SpO} 2<94 \%$ at admission $b$ & $8(57.1)$ & $15(14.3)$ & $\begin{array}{r}8.0 \\
(2.0-31.6)\end{array}$ \\
\hline Respiratory support ${ }^{b}$ & $12(85.7)$ & $25(23.8)$ & $\begin{array}{r}19.2 \\
(3.8-182.5)\end{array}$ \\
\hline Use of vasoactive drugs & $7(50.0)$ & $10(9.5)$ & $\begin{array}{r}19.5 \\
(2.3-38.5)\end{array}$ \\
\hline Need for intensive care ${ }^{b}$ & $12(85.7)$ & $26(24.8)$ & $\begin{array}{r}18.2 \\
(3.6-173.2)\end{array}$ \\
\hline Hospital stay $\geq 9 \mathrm{~d}$ & $7(87.5)$ & $43(50.0)$ & $\begin{array}{r}7 \\
(0.8-322.6)\end{array}$ \\
\hline
\end{tabular}

All values in no. (\%); ${ }^{a} P<0.05,{ }^{b} P=0.001$. 


\section{WHAT IS ALREADY KNOWN?}

- Neonates, male gender, pre-existing medical conditions, fever, lower respiratory tract infection, radiological changes suggestive of pneumonia or ARDS, and viral co-infection were associated with more severe course.

\section{WHAT THIS STUDY ADDS?}

- Children with underlying medical illnesses have significantly severe COVID-19 disease.

- Male gender, hypoxia (SpO2 <94\%) on admission, need for respiratory support, need for vasoactive drugs, ICU care, and length of hospital stay of $<10$ days is significantly associated with mortality.

Need for intensive care in our series is similar to that reported in literature [14]. Adult studies suggest presence of co-morbidities as an important predictor of need for intensive care[15], which was not found by us. Children requiring mechanical ventilation $(15.5 \%)$ were fewer than the cohort from USA $[6,16]$ as we had more nonrespiratory presentations.

A study of children from the European cohort concluded that neonates, male sex, pre-existing medical conditions, fever, lower respiratory tract infection, radiological changes of pneumonia or ARDS, and viral co-infection were associated with more severe course on univariate analysis; however, these were not correlated to mortality [11]. In our cohort, male sex, hypoxia ( $\mathrm{SpO} 2$ $<94 \%$ ) on admission, need for respiratory support, inotropes, intensive care, length of hospital stay $<10$ days was significantly associated with mortality. Male gender has been associated with a higher risk of severe disease and mortality because of higher ACE-2 receptor expression [17]. On regression analysis, $\mathrm{SpO} 2<94 \%$ on admission and length of hospital stay of $<10$ days were predictors of mortality and not the presence of comorbidities. This need to be corroborated with a bigger sample size. Experience from adult studies has shown mortality within 1 to 2 weeks of ICU admission [15].

As a retrospective study, certain important parameters like onset of symptoms from day of contact, source of infection, and exact duration of COVID-19 RTPCR positivity in all children could not be assessed.

To conclude, pediatric COVID-19 although considered a mild illness, children with co morbidity manifest with severe disease. Male sex, hypoxia on admission, need for intensive care, ventilator support, inotrope, hospital stay of $<10$ days are predictors of mortality. Policy of testing all admitted children for COVID-19 helps identify and segregate the cases, provide protocol based care, characterize the severity, initiate prompt treatment and improve outcome.

Contributors: SR,SSP,SBP: conceived, designed the study, finalised the manuscript; SR,VG,RM, LRD,SBP,SSP,MB: data collection, data analysis; SR,VG,RM,LRD,SSP,SBP: Literature search, interpretation of data, writing manuscript. All authors approved the final manuscript.

Ethics clearance: Institutional Ethics Committee Bai Jerbai Wadia Hospital for Children; No. IEC-BJWHC/ 89/2020, dated 26 August, 2020.

Funding: None; Competing interests: None stated.

\section{REFERENCES}

1. Directorate General of Health Services. Revised National Clinical Management Guideline for COVID-19. New Delhi (IN): Ministry of Health \& Family Welfare. Available at www.mohfw.gov.in.

2. National Centre for Disease Control. COVID -19 Outbreak Guidelines for Setting up. New Delhi (IN): Ministry of Health \& Family Welfare. Available at www.mohfw.gov.in.

3. Jiang L, Tang K, Levin M, et al. COVID-19 and multisystem inflammatory syndrome in children and adolescents. Lancet Infect Dis. 2020;20:e276-88.

4. Singh S, Jindal AK, Pilania RK. Diagnosis of Kawasaki disease. Int J Rheum Dis. 2018;21:36-44.

5. Shobhavat L, Solomon R, Rao S, et al. Multisystem inflammatory syndrome in children: Clinical features and management-Intensive care experience from a pediatric public hospital in Western India. Indian J Crit Care Med. 2020; 24: 1089-94.

6. Zachariah P, Johnson CL, Halabi KC, et al. Epidemiology, clinical features, and disease severity in patients with coronavirus disease 2019 (COVID-19) in a children's hospital in New York City, New York. JAMA Pediatr. 2020;174:e202430.

7. De Luca CD, Esposito E, Cristiani L, et al. Covid-19 in children: A brief overview after three months experience. Paediatr Respir Rev. 2020; 35:9-14.

8. Meena J, Yadav J, Saini L, Yadav A, Kumar J. Clinical features and outcome of sars-cov-2 infection in children: A systematic review and meta-analysis. Indian Pediatr. 2020;57: 820-26.

9. Dong Y, Mo X, Hu Y, et al. Epidemiology of COVID-19 among children in China. Pediatrics. 2020 ; 145:e20200702

10. Sarangi B, Reddy VS, Oswal JS, et al. Epidemiological and clinical characteristics of COVID-19 in Indian children in the initial phase of the pandemic: A cross-sectional study. Indian Pediatr. 2020; 57:914-17.

11. Götzinger F, Santiago-García B, Noguera-Julián A, et al. 
COVID-19 in children and adolescents in Europe: A multinational, multicentre cohort study. Lancet Child Adolesc Health. 2020;4:653-61.

12. Guo CX, He L, Yin JY, et al. Epidemiological and clinical features of pediatric COVID-19. BMC Med. 2020;18:250.

13. Yonker LM, Neilan AM, Bartsch Y, et al. Pediatric severe acute respiratory syndrome coronavirus 2 (SARS-CoV-2): Clinical presentation, infectivity, and immune responses. J Pediatr. 2020;227:45-52.

14. Kim L, Whitaker M, O'Halloran A, et al. Hospitalization rates and characteristics of children aged $<18$ years hospitalized with laboratory-confirmed COVID-19 COVID-NET, 14 States, March 1-July 25, 2020. Morb
Mortal Wkly Rep. 2020;69:1081-88.

15. Yang $\mathrm{X}, \mathrm{Yu} \mathrm{Y}, \mathrm{Xu} J$, et al. Clinical course and outcomes of critically ill patients with SARS-CoV-2 pneumonia in Wuhan, China: A single-centered, retrospective, observational study. Lancet Respir Med. 2020;8:475-81.

16. Shekerdemian LS, Mahmood NR, Wolfe KK, et al. Characteristics and outcomes of children with coronavirus disease 2019 (COVID-19) infection admitted to US and Canadian pediatric intensive care units. JAMA Pediatr. 2020;174:868-73.

17. Yuki K, Fujiogi M, Koutsogiannaki S. COVID-19 pathophysiology: A review. Clin Immunol. 2020;215: 108427. 